PROCEEDINGS OF THE

AMERICAN MATHEMATICAL SOCIETY

Volume 135, Number 4, April 2007, Pages 1097-1106

S 0002-9939(06)08549-2

Article electronically published on September 26, 2006

\title{
ON CHARACTERIZATION AND PERTURBATION OF LOCAL $C$-SEMIGROUPS
}

\author{
YUAN-CHUAN LI AND SEN-YEN SHAW
}

(Communicated by Joseph A. Ball)

\begin{abstract}
Let $S(\cdot)$ be a $\left(C_{0}\right)$-group with generator $-B$, and let $\{T(t) ; 0 \leq$ $t<\tau\}$ be a local $C$-semigroup commuting with $S(\cdot)$. Then the operators $V(t):=S(-t) T(t), 0 \leq t<\tau$, form a local $C$-semigroup. It is proved that if $C$ is injective and $A$ is the generator of $T(\cdot)$, then $A+B$ is closable and $\overline{A+B}$ is the generator of $V(\cdot)$. Also proved are a characterization theorem for local $C$-semigroups with $C$ not necessarily injective and a theorem about solvability of the abstract inhomogeneous Cauchy problem: $u^{\prime}(t)=A u(t)+C f(t), 0<$ $t<\tau ; u(0)=C x$.
\end{abstract}

\section{INTRODUCTION AND RESULT}

The aim of this paper is to present some theorems about characterization and perturbation of local $C$-semigroups and the solvability of the associated inhomogeneous Cauchy problem.

Let $X$ be a complex Banach space and let $B(X)$ be the Banach algebra of all bounded (linear) operators on $X$. When $0<\tau<\infty$ (resp. $\tau=\infty$ ), a family $\{T(t) ; 0 \leq t<\tau\}$ in $B(X)$ is called a local $C$-semigroup (resp. (global) $C$-semigroup) on $X$ if

(a) $T(\cdot) x:[0, \tau) \rightarrow X$ is continuous for each $x \in X$.

(b) $T(s+t) C=T(s) T(t)$ for all $0 \leq s, t, t+s<\tau$ and $T(0)=C$.

$C$-semigroups have been studied in many papers, among them are [1, 2], 3], [7, and [10. Local $C$-semigroups have been studied in [4, 6], 8], 9], [1], 12, [13.

When $\tau=\infty$ and $C=I$, the identity operator, $T(\cdot)$ is a classical $\left(C_{0}\right)$-semigroup. Thus $\left(C_{0}\right)$-semigroups form a subclass of the class of $C$-semigroups, and clearly, every $C$-semigroup can be viewed as a local $C$-semigroup defined on $[0, \tau)$ for any $0<\tau<\infty$. In general, a local $C$-semigroup on $[0, \tau)$ for some $\tau<\infty$ is not necessarily extendable to the whole half line $[0, \infty)$. Results concerning extension of local $C$-semigroups can be found in 4 and 12 .

We first state the following general characterization theorem for local $\mathrm{C}$ semigroups. Its proof will be given in Section 2.

Received by the editors August 8, 2005 and, in revised form, November 7, 2005.

2000 Mathematics Subject Classification. Primary 47D06, 47D60.

$K e y$ words and phrases. Local $C$-semigroup, $\left(C_{0}\right)$-group, generator, perturbation.

This research was supported in part by the National Science Council of Taiwan.

(C)2006 American Mathematical Society Reverts to public domain 28 years from publication 
Theorem 1. A strongly continuous family $\{T(t) ; 0 \leq t<\tau\}$ is a local C-semigroup if and only if $T(\cdot)$ commutes with $C$ and satisfies $T(0)=C$ and

$$
(1 * T)(s)[T(t)-C]=[T(s)-C](1 * T)(t) \text { for all } 0 \leq s, t, s+t<\tau .
$$

We are mainly interested in those local $C$-semigroups which are nondegenerate. A local $C$-semigroup $\{T(t) ; 0 \leq t<\tau\}$ will be said to be nondegenerate if one of the equivalent conditions in the next lemma is satisfied.

Lemma 2. The following statements are equivalent:

(c1) $C$ is injective.

(c2) $\lim T(t) x=0$ implies $x=0$.

(c3) $T(t) x \equiv 0$ on $\left(0, s_{1}\right)$ for some $s_{1} \in(0, \tau / 2)$ implies $x=0$.

(c4) $T(t) x \equiv 0$ on $(0, \tau / 2)$ implies $x=0$.

Proof. (c1) $\Rightarrow(\mathrm{c} 2)$. If $T(t) x \rightarrow 0$ as $t \rightarrow 0^{+}$, by (a) and (b) we have $C x=$ $\lim _{t \rightarrow 0^{+}} T(t) x=0$, and $(\mathrm{c} 1)$ implies $x=0$.

$(\mathrm{c} 2) \Rightarrow(\mathrm{c} 3) \Rightarrow(\mathrm{c} 4)$ are obvious.

(c4) $\Rightarrow(\mathrm{c} 1)$. If $C x=0$, then from (b) we see that $T(s) T(t) x=0$ for all $0<s, t<\tau / 2$, which implies $x=0$ by (c4). Hence $C$ is injective.

When $\tau=\infty$, the above definition of nondegeneracy coincides with the usual definition of nondegeneracy for $C$-semigroups, i.e., $T(t) x=0$ for all $t>0$ implies $x=0$. But, when $\tau<\infty,(\mathrm{c} 4)$ is even strictly stronger than the following condition: $\left(\mathrm{c}^{\prime}\right) T(t) x \equiv 0$ on $\left(0, s_{2}\right)$ for some $s_{2} \in(\tau / 2, \tau)$ implies $x=0$,

because, unlike the case $\tau=\infty,\left(\mathrm{c}^{\prime}\right)$ is not equivalent to the injectivity of $C$ when $\tau<\infty$. Also, unlike $C$-semigroups, a local $C$-semigroup $\{T(t) ; 0 \leq t<\tau\}$ with $\tau<\infty$ need not be commutative, although $C$ commutes with each $T(t), t \in[0, \tau)$, and $\{T(t) ; 0 \leq t<\tau / 2\}$ is a commutative subfamily, by (b). These interesting phenomena are illustrated by the following example.

Let $U:[\tau / 2, \tau) \rightarrow B(X)$ be a strongly continuous function such that $U(\tau / 2)=0$ and $U(t)$ is injective for all $t \in(\tau / 2, \tau)$. The operator $C:=0 \oplus I \in B(X \times X)$, with $I$ the identity operator on $X$, is not injective. We define $T:[0, \tau) \rightarrow B(X \times X)$ by

$$
T(t):= \begin{cases}0 \oplus I & \text { if } 0 \leq t<\tau / 2, \\ U(t) \oplus I & \text { if } \tau / 2 \leq t<\tau .\end{cases}
$$

Then $T(\cdot)$ is strongly continuous and satisfies

$$
T(s) T(t)=T(t) T(s)=0 \oplus I=T(s+t) C \text { for all } 0 \leq s, t, s+t<\tau .
$$

Hence this $T(\cdot)$ is a local $C$-semigroup with $C$ not injective. But, it satisfies condition $\left(\mathrm{c}^{\prime}\right)$. Indeed, if there is a $t \in(\tau / 2, \tau)$ such that $T(t) x=0$, then since

$$
C(t) x=(U(t) \oplus I)\left(x_{1}, x_{2}\right)=\left(U(t) x_{1}, x_{2}\right)
$$

and $U(t)$ is injective, we have $x_{1}=x_{2}=0$ and hence $x=0$. Moreover, since the family $\{U(t) ; \tau / 2 \leq t<\tau\}$ need not be commutative, the above local $C$-semigroup $T(\cdot)$ may not be a commutative family.

When $C$ is injective, $T(\cdot)$ is indeed commutative (see [8, Lemma 1.1]).

Now for a nondegenerate local $C$-semigroup $T(\cdot)$, by either (c2) or (c4) one can define the generator $A$ by

(d) $\quad x \in D(A)$ and $A x=y \Leftrightarrow \int_{0}^{t} T(s) y d s=T(t) x-C x$ for all $t \in[0, \tau)$. 
It is known [8] that this definition is equivalent to the one in the sense of Da Prato 1]:

$$
\left\{\begin{array}{l}
D(A)=\left\{x \in X ; \lim _{h \rightarrow 0^{+}}(T(h) x-C x) / h \in R(C)\right\} \\
A x=C^{-1} \lim _{h \rightarrow 0^{+}}(T(h) x-C x) / h \text { for } x \in D(A)
\end{array}\right.
$$

The following characterization of a nondegenerate local $C$-semigroup is proved in [8. Lemma 2.1 and Proposition 2.2].

Proposition 3. Let $C \in B(X)$ be an injection and let $\{T(t) ; 0 \leq t<\tau\}$ be a strongly continuous family of operators on $X$.

(i) If $T(\cdot)$ is a local $C$-semigroup with generator $A$, then $A$ is closed and satisfies $C^{-1} A C=A, R\left(\int_{0}^{t} T(s) d s\right) \subset D(A)$ and

$$
T(t) x-C x= \begin{cases}A \int_{0}^{t} T(s) x d s & \text { for } x \in X \\ \int_{0}^{t} T(s) A x d s & \text { for } x \in D(A)\end{cases}
$$

for all $t \in[0, \tau)$.

(ii) If $A$ is a closed operator satisfying $R\left(\int_{0}^{t} T(s) d s\right) \subset D(A)$ and $(1.2)$, then $T(\cdot)$ is a local $C$-semigroup with generator $C^{-1} A C$.

Consequently, a strongly continuous family $\{T(t) ; 0 \leq t<\tau\}$ is a local $C$ semigroup with generator $A$ if and only if $A$ is closed and satisfies $C^{-1} A C=A$, $R\left(\int_{0}^{t} T(s) d s\right) \subset D(A)$ and $(1.2)$.

The following characterization of generator in terms of solvability of the abstract Cauchy problem is proved in [8, Corollary 2.6].

Proposition 4. An operator $A$ is the generator of a local $C$-semigroup $\{T(t) ; t \in$ $[0, \tau)\}$ if and only if the abstract Cauchy problem

$\operatorname{ACP}(A ; C x+C(1 * g), 0) \quad\left\{\begin{array}{l}u^{\prime}(t)=A u(t)+C x+\int_{0}^{t} C g(s) d s, \quad 0<t<\tau, \\ u(0)=0\end{array}\right.$

has a unique solution for every $x \in X$ and $g \in L_{\text {loc }}^{1}([0, \tau), X)$. The solution is $u(t)=\int_{0}^{t} T(s) x d s+\int_{0}^{t} \int_{0}^{s} T(s-r) g(r) d r d s$.

This proposition can be used to deduce assertion (ii) of the next theorem, which is concerned with the solvability of the abstract Cauchy problem

$$
\operatorname{ACP}(A ; C f, C x) \quad\left\{\begin{array}{l}
u^{\prime}(t)=A u(t)+C f(t), \quad 0<t<\tau, \\
u(0)=C x
\end{array}\right.
$$

for suitable vector $x$ and vector function $f$.

Theorem 5. Let $A$ be the generator of a nondegenerate local $C$-semigroup $\{T(t)$; $0 \leq t<\tau\}$ on $X, x \in X$, and $f \in C([0, \tau), X)$.

(i) If $\operatorname{ACP}(A ; C f, C x)$ has a strong solution $u$, then $u \equiv T(\cdot) x+T * f$.

(ii) If $u:=T(\cdot) x+T * f \in C([0, \tau),[D(A)])$, where $[D(A)]$ is the Banach space $D(A)$ equipped with the graph norm, then $u$ is a strong solution of $\operatorname{ACP}(A ; C f, C x)$.

(iii) If either $f \in C^{1}([0, \tau), X)$ or $f \in C([0, \tau),[D(A)])$, then for every $x \in D(A)$ $\operatorname{ACP}(A ; C f, C x)$ has the unique strong solution $u(t):=T(t) x+(T * f)(t), 0 \leq t<\tau$. 
Theorem 5 is well known for the case $\tau=\infty$ (cf. [7, Theorem 7.1 and Corollary 7.5]) and the case $C=I$ (cf. [5]).

As an application of Proposition 4, it is deduced in [9, Theorem 2] that if $A$ is the generator of a local $C$-semigroup and if $B \in B(X)$ satisfies $R(B) \subset R(C)$ and $B C x=C B x$ for $x \in D(A)$, then $A+B$ also generates a local $C$-semigroup. In the following theorem we extend this result to those unbounded perturbation operators $B$ that generate some commuting $\left(C_{0}\right)$-groups.

Theorem 6. Let $T(\cdot)$ be a local $C$-semigroup on a Banach space $X$ and let $S(\cdot)$ be a $\left(C_{0}\right)$-group with generator $-B$. Suppose $S(t) T(s)=T(s) S(t)$ for all $0 \leq s, t<\tau$. Let $V(t):=S(-t) T(t)$ for $t \in[0, \tau)$. Then

(i) $\{V(t) ; t \in[0, \tau)\}$ is the unique local $C$-semigroup commuting with $S(\cdot)$ and $T(\cdot)$ and satisfying

$$
\int_{0}^{t} S(u)(1 * V)(u) d u=\int_{0}^{t} S(u)(1 * T)(t-u) d u .
$$

(ii) If $C$ is injective and $A$ is the generator of $T(\cdot)$, then $A+B$ is closable and $\overline{A+B}$ is the generator of $V(\cdot)$. In particular, if $B \in B(X)$ commutes with $T(\cdot)$, then $A+B$ generates the local $C$-semigroup $\left\{e^{-t B} T(t) ; 0 \leq t<\tau\right\}$.

We remark that a multiplicative perturbation theorem for local $C$-semigroups has been proved in [13].

The proofs of Theorems 1, 5, and 6 will be given in Sections 2 and 3, and a simple example of an application of Proposition 4 and Theorems 5 and 6 will be given in Section 4.

\section{Proofs of Theorems 1 And 5}

Proof of Theorem 1. Suppose $T(\cdot)$ is a local $C$-semigroup on $X$. Then, by the commutativity of $(1 * T)(t)$ with $T(s)$ and $C$ for $0 \leq s, t, s+t<\tau$, we have

$$
\begin{aligned}
T(s)(1 * T)(t) x & =\int_{0}^{t} T(s) T(u) x d u=\int_{0}^{t} T(s+u) C x d u=\int_{s}^{s+t} T(u) C x d u \\
& =\int_{t}^{s+t} T(u) C x d u+\int_{0}^{t} T(u) C x d u-\int_{0}^{s} T(u) C x d u \\
& =(1 * T)(s) T(t) x+C(1 * T)(t) x-(1 * T)(s) C x
\end{aligned}
$$

for $x \in X$ and $0 \leq s, t, s+t<\tau$. This shows (1.1).

Conversely, suppose that $T(\cdot)$ satisfies (1.1). For fixed $s, t \in[0, \tau)$ with $s+t<\tau$, we replace $s$ and $t$ in (1.1) by $s+t-r$ and $r$, respectively, to obtain

$(1 * T)(s+t-r) T(r) x-T(s+t-r)(1 * T)(r) x=(1 * T)(s+t-r) C x-C(1 * T)(r) x$ for all $x \in X$ and $r \in[0, s+t)$. By integrating the right-hand side with respect to $r$ from 0 to $t$, we obtain from $C T(\cdot)=T(\cdot) C$ that

$$
\begin{aligned}
\int_{0}^{t}(1 & * T)(s+t-r) C x d r-\int_{0}^{t} C(1 * T)(r) x d r \\
& =\int_{s}^{s+t}(1 * T)(r) C x d r-\int_{0}^{t} C(1 * T)(r) x d r \\
& =\left(\int_{0}^{s+t}-\int_{0}^{s}-\int_{0}^{t}\right)(1 * T)(r) C x d r .
\end{aligned}
$$


On the other hand, from the left-hand side we have

$$
\begin{aligned}
& \int_{0}^{t}(1 * T)(s+t-r) T(r) x d r-\int_{0}^{t} T(s+t-r)(1 * T)(r) x d r \\
&=\left.(1 * T)(s+t-r)(1 * T)(r) x\right|_{0} ^{t}+\int_{0}^{t} T(s+t-r)(1 * T)(r) x d r \\
&-\int_{0}^{t} T(s+t-r)(1 * T)(r) x d r \\
&=(1 * T)(s)(1 * T)(t) x-(1 * T)(s+t)(1 * T)(0) x \\
&=(1 * T)(s)(1 * T)(t) x .
\end{aligned}
$$

Therefore $(1 * T)(\cdot)$ satisfies

$$
(1 * T)(s)(1 * T)(t) x=\left(\int_{0}^{s+t}-\int_{0}^{s}-\int_{0}^{t}\right)(1 * T)(r) C x d r
$$

for all $x \in X$ and $s, t, s+t \in[0, \tau)$. By differentiation, it is clear that $T(\cdot)$ is a local $C$-semigroup.

Proof of Theorem 5. (i) Suppose $u$ is a strong solution of $\operatorname{ACP}(A ; C f, C x)$. Then $u(0)=C x$ and $u^{\prime}=A u+C f$, so that $u-C x=1 * u^{\prime}=1 *(A u+C f)$. Therefore we have

$$
\begin{aligned}
T * u-C(1 * T)(\cdot) x & =T *(u-C x)=(1 * T) A * u+1 *(T * C f) \\
& =(T-C) * u+C(1 * T) * f \\
& =T * u-C(1 * u)+C 1 *(T * f) .
\end{aligned}
$$

Since $C$ is injective, we have $1 * u=(1 * T)(\cdot) x+1 *(T * f)$. By differentiation, we have $u=T(\cdot) x+T * f$. This also proves that the strong solution of $\operatorname{ACP}(A ; C f, C x)$ is unique.

(ii) Suppose $u:=T(\cdot) x+T * f \in C([0, \tau),[D(A)])$. Then $u(0)=C x, A u \in$ $C([0, \tau), X)$ and $1 * u=(1 * T)(\cdot) x+(1 * T * f)$, so that, by the closedness of $A$, we have

$$
1 *(A u)=A(1 * u)=T(\cdot) x-C x+(T-C) * f=u-C x-C(1 * f) .
$$

By differentiation, we have $u^{\prime}=A u+C f$.

Derivation of (ii) from Proposition 4. By Proposition 4, $\operatorname{ACP}(A ; C x+C(1 * f), 0)$ has a unique solution $v$ given by $v(t)=\int_{0}^{t} u(s) d s$ for $t \in[0, \tau)$, where $u=$ $T(\cdot) x+T * f$. If $u \in C([0, \tau),[D(A)])$, then by the closedness of $A$, we have $v(t) \in D(A)$ and $A v(t)=\int_{0}^{t} A u(s) d s$ is continuously differentiable in $X$ on $[0, \tau)$. Hence $u=v^{\prime}=A v+C x+C(1 * f)$ is continuously differentiable and satisfies $u^{\prime}(t)=A u(t)+C f(t), 0<t<\tau$, and $u(0)=T(0) x=C x$, i.e., $u$ is a strong solution of $\operatorname{ACP}(A ; C f, C x)$.

(iii) Consider the following two cases.

If $f \in C^{1}([0, \tau), X)$, then $T * f \in C^{1}([0, \tau), X)$ so that

$$
\begin{aligned}
A[1 *(T * f)] & =A(1 * T) * f=(T-C) * f \\
& =T * f-C(1 * f) \in C^{1}([0, \tau), X) .
\end{aligned}
$$

Since $A$ is closed, it follows from differentiation that $(T * f)(t) \in D(A)$ for all $t \in[0, \tau)$ and $A(T * f)=(T * f)^{\prime}-C f \in C([0, \tau), X)$, i.e., $T * f \in C([0, \tau),[D(A)])$. 
If $f \in C([0, \tau),[D(A)])$, then $A(T * f)=T * A f \in C([0, \tau), X)$, and hence we also have $T * f \in C([0, \tau),[D(A)])$.

Since $T(\cdot) x \in C([0, \tau),[D(A)])$ for $x \in D(A)$, in both the above cases, we have $u:=T(\cdot) x+T * f \in C([0, \tau),[D(A)])$ for every $x \in D(A)$. Therefore $u$ is a strong solution of $\operatorname{ACP}(A ; C f, C x)$ for every $x \in D(A)$, by (ii).

\section{Proof of Theorem 6}

We first prove the following proposition.

Proposition 7. Let $T(\cdot)$ and $S(\cdot)$ be two commuting local $C$-semigroups with generators $A$ and $B$, respectively. Then the following hold.

(i) $A+B$ is closable and satisfies:

$$
(A+B) \subset C^{-1}(A+B) C \text { and } \overline{A+B} \subset C^{-1} \overline{A+B} C .
$$

(ii) If, in addition, either one of $T(\cdot)$ and $S(\cdot)$ is even a $\left(C_{0}\right)$-semigroup, then

$$
C^{-1} \overline{A+B} C=\overline{A+B} \text {. }
$$

Proof. (i) First, we show that $A+B$ is closable. Let $\left\{x_{n}\right\}$ be a null sequence in $D(A+B)$ such that $(A+B) x_{n}$ converges to a vector $y \in X$. We need to show $y=0$. Observe that $S(t) T(s)=T(s) S(t)$ implies that $S(t) A x=A S(t) x$ for $x \in D(A)$. Hence we have

$$
\begin{aligned}
(1 * T)(t)(1 * S)(s) y & =\lim _{n \rightarrow \infty}(1 * T)(t)(1 * S)(s)(A+B) x_{n} \\
& =\lim _{n \rightarrow \infty}\left\{[T(t)-C](1 * S)(s) x_{n}+(1 * T)(t)[S(s)-C] x_{n}\right\} \\
& =[T(t)-C](1 * S)(s) 0+(1 * T)(t)[S(s)-C] 0=0
\end{aligned}
$$

and then $T(t) S(s) y=0$ for all $s, t \in(0, \tau)$, by differentiation. Then the strong continuity of $T(\cdot)$ and $S(\cdot)$ at 0 implies $C^{2} y=0$ and hence $y=0$. Therefore $A+B$ is closable.

Let $x \in D(A+B)=D(A) \cap D(B)$. Since $A$ and $B$ are generators, by Proposition 3(i), we have $C^{-1} A C x=A x$ and $C^{-1} B C x=B x$, so that $C x \in D(A) \cap D(B)=$ $D(A+B)$ and $A C x=C A x$ and $B C x=C B x$. Hence $(A+B) C x=A C x+B C x=$ $C(A+B) x$ and so $x \in D\left(C^{-1}(A+B) C\right)$ and $(A+B) x=C^{-1}(A+B) C x$. Hence $(A+B) \subset C^{-1}(A+B) C$. Next, we show $\overline{A+B} \subset C^{-1} \overline{A+B} C$. If $x \in D(\overline{A+B})$, then there is a sequence $\left\{x_{n}\right\}$ in $D(A+B)$ such that $\left(x_{n},(A+B) x_{n}\right) \rightarrow(x, \overline{A+B} x)$. As above, we have $(A+B) C x_{n}=C(A+B) x_{n} \rightarrow C \overline{A+B} x$. This with the fact that $C x_{n} \rightarrow C x$ implies that $C x \in D(\overline{A+B})$ and $\overline{A+B} C x=C \overline{A+B} x$, or $\overline{A+B} x=C^{-1} \overline{A+B} C x$. Therefore $\overline{A+B} \subset C^{-1} \overline{A+B} C$.

(ii) Assume $S(\cdot)$ is a $\left(C_{0}\right)$-semigroup. It remains to show the inclusion:

$$
C^{-1} \overline{A+B} C \subset \overline{A+B} .
$$

Let $x \in D\left(C^{-1} \overline{A+B} C\right)$ and $y:=C^{-1} \overline{A+B} C x$. Then $C y=\overline{A+B} C x$. So, there is a sequence $\left\{z_{n}\right\}$ in $D(A+B)$ such that $\left(z_{n},(A+B) z_{n}\right) \rightarrow(C x, C y)$ strongly as $n \rightarrow \infty$. Therefore we have for every $s, t \in[0, \tau)$

$$
\begin{aligned}
& (1 * T)(s)(1 * S)(t) C y \\
& =\lim _{n \rightarrow \infty}(1 * T)(s)(1 * S)(t)(A+B) z_{n} \\
& =\lim _{n \rightarrow \infty}\left[(1 * S)(t)[T(s)-C] z_{n}+(1 * T)(s)(S(t)-I) z_{n}\right] \\
& =(1 * S)(t)[T(s)-C] C x+(1 * T)(s)(S(t)-I) C x .
\end{aligned}
$$


Since $T(\cdot), S(\cdot)$, and $C$ commute, it follows from the injectivity of $C$ that

$$
(1 * T)(s)[(1 * S)(t) y-(S(t)-I) x]=[T(s)-C](1 * S)(t) x
$$

for every $s, t \in[0, \tau)$. By the definition of generator, this implies that $(1 * S)(t) x \in$ $D(A)$ and

$$
A(1 * S)(t) x=(1 * S)(t) y-(S(t)-I) x=(1 * S)(t) y-B(1 * S)(t) x .
$$

Hence we have for every $t \in[0, \tau)$

$$
(1 * S)(t) y=(A+B)(1 * S)(t) x=\overline{A+B}(1 * S)(t) x .
$$

By differentiation, we have $S(t) x \in D(\overline{A+B})$ and $S(t) y=\overline{A+B} S(t) x$. Since $S(0)=I$, this implies that $x \in D(\overline{A+B})$ and $y=\overline{A+B} x$. Therefore $C^{-1} \overline{A+B} C$ $\subset \overline{A+B}$.

Proof of Theorem 6. (i) Since $S(\cdot)$ commutes with $T(\cdot)$, clearly $V(\cdot)$ is a local $C$ semigroup commuting with $S(\cdot)$ and $T(\cdot) . V(\cdot)$ satisfies (1.3):

$$
\begin{aligned}
\int_{0}^{t} S(u)(1 * V)(u) d u & =\int_{0}^{t} S(u) \int_{0}^{u} S(-s) T(s) d s d u=\int_{0}^{t} \int_{s}^{t} S(u-s) T(s) d u d s \\
& =\int_{0}^{t} \int_{0}^{t-s} S(u) T(s) d u d s=\int_{0}^{t} S(u) \int_{0}^{t-u} T(s) d s d u \\
& =\int_{0}^{t} S(u)(1 * T)(t-u) d u .
\end{aligned}
$$

Suppose $V_{1}(\cdot)$ and $V_{2}(\cdot)$ are two functions satisfying (1.3). Then the function $V(\cdot):=$ $V_{1}(\cdot)-V_{2}(\cdot)$ satisfies $\int_{0}^{t} S(u)(1 * V)(u) d u=0$ for all $t \in[0, \tau)$. Hence $S(t)(1 * V)(t)=$ 0 for all $t \in[0, \tau)$. Since $S(t)$ is injective, we must have $V(\cdot) \equiv 0$.

(ii) Since $S(-t)$ is a $\left(C_{0}\right)$-semigroup with generator $B$, an application of Proposition 7 (with $S(t)$ therein replaced by $S(-t)$ ) yields that $A+B$ is closable and $C^{-1} \overline{A+B} C=\overline{A+B}$.

Since $(1 * T)(t) A \subset A(1 * T)(t)=T(t)-C$ for $t \in[0, \tau)$, and since $A$ is closed and $S(t) A y=A S(t) y$ for $y \in D(A)$ we have $R\left(\int_{0}^{t} S(u)(1 * T)(t-u) d u\right) \subset D(A)$ and

$$
\begin{aligned}
\int_{0}^{t} S(u)(1 * T)(t-u) d u A & \subset A \int_{0}^{t} S(u)(1 * T)(t-u) d u \\
& =\int_{0}^{t} S(u) A(1 * T)(t-u) d u \\
& =\int_{0}^{t} S(u)[T(t-u)-C] d u \\
& =\frac{d}{d t} \int_{0}^{t} S(u)(1 * T)(t-u) d u-\int_{0}^{t} S(u) C d u \\
& =\frac{d}{d t} \int_{0}^{t} S(u)(1 * V)(u) d u-\int_{0}^{t} S(u) C d u \\
& =S(t)(1 * V)(t)-\int_{0}^{t} S(u) C d u
\end{aligned}
$$


for all $t \in[0, \tau)$. This and (1.3) imply that

$$
\begin{aligned}
\int_{0}^{t} S(u)(1 * V)(u) A d u & \subset A \int_{0}^{t} S(u)(1 * V)(u) d u \\
& =S(t)(1 * V)(t)-\int_{0}^{t} S(u) C d u .
\end{aligned}
$$

Differentiating (1.3), we obtain

$$
S(t)(1 * V)(t)=\int_{0}^{t} S(u) T(t-u) d u \text { for all } t \in[0, \tau) .
$$

Since $1 * V$ commutes with $S(\cdot)$, it commutes with the generator $-B$, i.e., $(1 * V)(u) x \in D(B)$ and $B(1 * V)(u) x=(1 * V)(u) B x$ for $x \in D(B)$, so that $S^{\prime}(u)(1 * V)(u) x=-B S(u)(1 * V)(u) x=-S(u) B(1 * V)(u) x=-S(u)(1 * V)(u) B x$ for all $u \in[0, \tau)$. Then, using integration by parts, the closedness of $B$, and (1.3), we obtain for $x \in D(B)$ and $t \in[0, \tau)$,

$$
\begin{aligned}
S(t)(1 * V)(t) x & =-\int_{0}^{t} S(u)(1 * V)(u) B x d u+\int_{0}^{t} S(u) V(u) x d u \\
& =-\int_{0}^{t} B S(u)(1 * V)(u) x d u+\int_{0}^{t} S(u) V(u) x d u \\
& =-B \int_{0}^{t} S(u)(1 * T)(t-u) x d u+\int_{0}^{t} S(u) V(u) x d u .
\end{aligned}
$$

Combining this and (3.2), and by the closedness of $B$ again, we obtain

$$
\begin{aligned}
\int_{0}^{t} S(u)(1 * T)(t-u) d u B & \subset B \int_{0}^{t} S(u)(1 * T)(t-u) d u \\
& =-\int_{0}^{t} S(u) T(t-u) d u+\int_{0}^{t} S(u) V(u) d u
\end{aligned}
$$

for every $t \in[0, \tau)$.

Now we obtain from (1.3), (3.2) and (3.3) that

$$
\begin{aligned}
\int_{0}^{t} S(u)(1 * V)(u) B d u & \subset B \int_{0}^{t} S(u)(1 * V)(u) d u \\
& =\int_{0}^{t} S(u) V(u) d u-S(t)(1 * V)(t) .
\end{aligned}
$$

Hence, summing (3.1) and (3.4) and then taking the closure of $A+B$ we have for every $t \in[0, \tau)$

$$
\begin{aligned}
\int_{0}^{t} S(u)(1 * V)(u)(\overline{A+B}) d u & \subset(\overline{A+B}) \int_{0}^{t} S(u)(1 * V)(u) d u \\
& =\int_{0}^{t} S(u)[V(u)-C] d u .
\end{aligned}
$$

Since $\overline{A+B}$ is closed, differentiation with respect to $t$ yields

$$
R(S(t)(1 * V)(t)) \subset D(\overline{A+B})
$$

and

$$
S(t)(1 * V)(t) \overline{A+B} \subset \overline{A+B} S(t)(1 * V)(t)=S(t)[V(t)-C]
$$


for all $t \in[0, \tau)$. Since $S(t)$ is injective, $(1 * V)(t) \overline{A+B} \subset V(t)-C$. On the other hand, since $S(\cdot)$ commutes with $V(\cdot)$, we have $R((1 * V)(t) S(t)) \subset D(\overline{A+B})$ and $\overline{A+B}(1 * V)(t) S(t)=[V(t)-C] S(t)$. Then, by the surjectivity of $S(t)$, we obtain that $R((1 * V)(t)) \subset D(\overline{A+B})$ and

$$
\overline{A+B}(1 * V)(t)=V(t)-C .
$$

Thus we have shown

$$
(1 * V)(t) \overline{A+B} \subset \overline{A+B}(1 * V)(t)=V(t)-C .
$$

The conclusion of the theorem now follows from Proposition 3.

\section{An illustrative eXAmple}

Consider the following initial value problems in $c_{0}$ :

$$
\begin{aligned}
& \left\{\begin{array}{l}
v_{n}^{\prime}(t)=n(1+i n) u_{n}(t)+e^{-n} q_{n}+\int_{0}^{t} e^{-n} g_{n}(s) d s, 0<t<1, \\
v_{n}(0)=0,
\end{array}\right. \\
& \left\{\begin{array}{l}
u_{n}^{\prime}(t)=n(1+i n) u_{n}(t)+e^{-n} f_{n}(t), 0<t<1, \\
u_{n}(0)=e^{-n} q_{n} .
\end{array}\right.
\end{aligned}
$$

The family $\{T(t) ; 0 \leq t \leq 1\}$, defined by $T(t) x:=\left(e^{-n} e^{n t} x_{n}\right), x=\left(x_{n}\right) \in$ $c_{0}, 0 \leq t \leq 1$, is a local $C$-semigroup with $C:=\bigoplus_{n=1}^{\infty} e^{-n} \in B\left(c_{0}\right)$ and with generator $A:=\bigoplus_{n=1}^{\infty} n$. The diagonal matrix $-B:=-i A^{2}=\bigoplus_{n=1}^{\infty}\left(-i n^{2}\right)$ generates the $C_{0}$-group $S(\cdot)$, defined by $S(t) x:=\left(e^{-i n^{2} t} x_{n}\right), x=\left(x_{n}\right) \in c_{0}, t \geq 0$, which commutes with $T(\cdot)$. By Theorem $6, \overline{A+B}$ generates the local $C$-semigroup $\{V(t) ; 0 \leq t<1\}$, defined by $V(t) x:=\left(e^{-n} e^{n(1+i n) t} x_{n}\right), x=\left(x_{n}\right) \in c_{0}$.

If, for instance, $g(t)=\left(g_{n}(t)\right) \in c_{0}$ for all $t \in[0,1)$ and the functions $\left\{g_{n}\right\}$ are uniformly continuous on $[0,1)$, then $g \in C\left([0,1) ; c_{0}\right)$. Now it follows from Proposition 4 that, for any $q \in c_{0}$, (4.1) has a unique solution $v \in C\left([0,1) ; c_{0}\right)$, which is given by

$$
\begin{aligned}
v(t) & =\int_{0}^{t} V(s) q d s+\int_{0}^{t} \int_{0}^{s} V(s-r) g(r) d r d s \\
& =\left(e^{-n}\left[\frac{1}{n(1+i n)}\left(e^{n(1+i n) t}-1\right) q_{n}+\int_{0}^{t} \int_{0}^{s} e^{n(1+i n)(s-r)} g_{n}(r) d r d s\right]\right),
\end{aligned}
$$

$0 \leq t<1$.

If $\left(n^{2} f_{n}(t)\right) \in c_{0}$ for all $t \in[0,1)$ and the functions $\left\{n^{2} f_{n}\right\}$ are uniformly continuous on $[0,1)$, then $f \in C([0,1) ;[D(\overline{A+B})])$. It follows from Theorem 5 that, for any $q \in c_{0}$ with $\lim _{n \rightarrow \infty} n^{2} q_{n}=0,(4.2)$ has a unique solution $u \in C\left([0,1) ; c_{0}\right)$, which is given by

$$
\begin{aligned}
u(t) & =V(t) q+\int_{0}^{t} V(t-s) g(s) d s \\
& =\left(e^{-n}\left[e^{n(1+i n) t} q_{n}+\int_{0}^{t} e^{n(1+i n)(t-s)} f_{n}(s) d s\right]\right), 0 \leq t<1 .
\end{aligned}
$$




\section{REFERENCES}

[1] G. Da Prato, Semigruppi regolarizzabili, Recerche di Mat. 15 (1966), 223-248. MR0225199 $(37: 793)$

[2] E. B. Davies and M. M. Pang, The Cauchy problem and a generalization of the Hille-Yosida theorem, Proc. London Math. Soc. 55 (1987), 181-208. MR0887288 (88e:34100)

[3] R. deLaubenfels, C-semigroups and the Cauchy problem, J. Funct. Anal. 111 (1993), 44-61. MR1200635 (94b:47055)

[4] M. Gao, Local $C$-semigroups and local $C$-cosine functions, Acta Math. Sci. 19 (1999), 201213. MR.1712307 (2000i:47083)

[5] J. A. Goldstein, Semigroups of Linear Operators and Applications, Oxford University Press, 1985. MR.0790497 (87c:47056)

[6] C.-C. Kuo and S.-Y. Shaw, Abstract Cauchy problems associated with local C-semigroups, in Semigroups of Operators: Theory and Applications: Second International Conference, Rio de Janeiro, Brazil, September 10-14, 2001 (SOTA2), Ed. by C. Kubrusly, N. Levan, and M. da Silveira, Optimization Software Inc., Publications, New York - Los Angeles, 2002, 158-168. MR2013626 (2004j:47084)

[7] Y.-C. Li and S.-Y. Shaw, $N$-times integrated $C$-semigroups and the abstract Cauchy problem, Taiwanese J. Math. 1 (1997), 75-102. MR1435499 (98g:47033)

[8] S.-Y. Shaw and C.-C. Kuo, Generation of local $C$-semigroups and solvability of the abstract Cauchy problems, Taiwanese J. Math., 9 (2005), 291-311. MR2142579|(2006a:47064)

[9] S.-Y. Shaw, C.-C. Kuo, and Y.-C. Li, Perturbation of local $C$-semigroups, Nonlinear Analysis 63 (2005), e2569-e2574.

[10] N. Tanaka and I. Miyadera, $C$-semigroups and the abstract Cauchy problem, J. Math. Anal. Appl. 170 (1992), 196-206. MR 1184734 (93j:47061)

[11] N. Tanaka and N. Okazawa, Local $C$-semigroups and local integrated semigroups, Proc. London Math. Soc. (3) 61 (1990), 63-90. MR.1051099 (91b:47093)

[12] S. W. Wang and M. C. Gao, Automatic extensions of local regularized semigroups and local regularized cosine functions, Proc. Amer. Math. Soc. 127 (1999), 1651-1663. MR.1600157 (99i:47072)

[13] T.-J. Xiao, J. Liang, and F. Li, A perturbation theorem of Miyadera type for local $C$ regularized semigroups, Taiwanese J. Math., 10 (2006), 153-162. MR2186169(2006h:47066)

Department of Applied Mathematics, National Chung-Hsing University, Taichung, 402 TAIWAN

E-mail address: ycli@amath.nchu.edu.tw

Graduate School of Engineering, Lunghwa University of Science and Technology, Gueishan, TaOYUan, 333 TaIWAN

E-mail address: shaw@math.ncu.edu.tw 